\title{
Variability of High-Luminosity Stars
}




\title{
RV Tauri Stars and Type II Cepheids in the Magellanic Clouds - Results from the MACHO Database
}

\author{
Karen R. Pollard 1 , C. Alcock 2,3 , R. A. Allsman 4 , D. Alves $2,4,5$ \\ T. S. Axelrod ${ }^{6}$, A. C. Becker ${ }^{7}$, D. P. Bennett ${ }^{2,3,8}$, K. H. Cook ${ }^{2,3}$, \\ K. C. Freeman ${ }^{6}$, K. Griest ${ }^{3,9}$, M. J. Lehner ${ }^{3,10}$, S. L. Marshall ${ }^{2,3,11}$, \\ B. A. Peterson ${ }^{6}$, M. R. Pratt ${ }^{3,7,12}$, P. J. Quinn ${ }^{13}$, W. Sutherland ${ }^{14}$, \\ A. Tomaney ${ }^{7}$, D. L. Welch ${ }^{15}$ (The MACHO Collaboration)
}

\begin{abstract}
A review of the properties of Type II Cepheids and RV Tauri stars in the Magellanic Clouds is presented. In the behaviour of their light and colour curves, the RV Tauri stars appear to be a direct extension of the Type II Cepheids to longer periods. A single $P-L-C$ relationship describes both the Type II Cepheids and RV Tauri stars in the LMC. The derived high intrinsic magnitudes for the RV Tauri variables supports the proposition that these objects are luminous stars evolving off the AGB. Preliminary analysis of the long time-series MACHO photometry indicates one star $\left(\mathrm{MACHO}^{*} 05: 37: 45.0-69: 54: 16\right)$ has an obvious 'period-quadrupled' periodicity, which is supporting evidence for a period-doubling bifurcation transition to chaotic pulsations.
\end{abstract}

\section{Introduction}

Type II Cepheids and RV Tauri stars are low-mass $\left(\sim 0.5-0.8 M_{\odot}\right)$ semiregular pulsating variables of the intermediate disk or halo population. Type II Cepheids generally display more regular pulsations and are smaller, less luminous supergiants. They are believed to be hydrogen- and helium-shell burning stars that

\footnotetext{
${ }^{1}$ Department of Physics and Astronomy, University of Canterbury, Private Bag 4800, Christchurch 8020, New Zealand

${ }^{2}$ Lawrence Livermore National Laboratory, Livermore, CA 94550, USA

${ }^{3}$ Center for Particle Astrophysics, University of California, Berkeley, CA 94720, USA

${ }^{4}$ Supercomputing Facility, Australian National University, Canberra, ACT 0200, Australia

${ }^{5}$ Department of Physics, University of California, Davis, CA 95616, USA

${ }^{6}$ Mt. Stromlo and Siding Spring Observatories, Australian National University, Weston Creek, ACT 2611, Australia

${ }^{7}$ Departments of Astronomy and Physics, University of Washington, Seattle, WA 98195, USA

${ }^{8}$ Department of Physics, University of Notre Dame, South Bend, IN 46556, USA

${ }^{9}$ Department of Physics, University of California, San Diego, La Jolla, CA 92093, USA

${ }^{10}$ Department of Physics, University of Sheffield, Sheffield, S3 7RH, U.K.

${ }^{11}$ Department of Physics, University of California, Santa Barbara, CA 93106, USA

${ }^{12}$ LIGO Project, MIT, Rm 20B-145, Cambridge, MA 02139, USA

${ }^{13}$ European Southern Observatory, Karl-Schwarzschild Str. 2, D-85748, Garching, Germany

${ }^{14}$ Department of Physics, University of Oxford, Oxford OX13RH, U.K.

${ }^{15}$ Department of Physics \& Astronomy, McMaster University, Hamilton, Ontario, Canada L8\$ $4 \mathrm{M} 1$
} 
are making blue loop excursions into the instability strip from the AGB. The RV Tauri stars are higher luminosity objects $\left(10^{3}-10^{4} L_{\odot}\right)$ which are believed to be in a more advanced stage of evolution at the end of the asymptotic giant branch. As luminous low-mass stars of the intermediate disk and halo population, RV Tauri stars are useful probes of our Galaxy's halo and bulge as well as the outer regions of nearby galaxies.

Of fundamental importance to our understanding of the RV Tauri variables and their relationship to the Type II Cepheids is a knowledge of their physical properties. In particular, our understanding of their evolutionary state, mass and pulsational behaviour (period-luminosity relationship, modes of pulsation, etc.) depends critically on their luminosities which have been poorly known until recently. Recent analysis of the MACHO project photometry of LMC variables (Pollard et al. 1997; Alcock et al. 1998) has resulted in the discovery of thirteen RV Tauri stars and a definitive assessment of the intrinsic luminosities of these stars and twenty Type II Cepheids.

\section{Pulsations in Type I and Type II Cepheids}

Type I Cepheids are arguably the most thoroughly understood intrinsic variable from a theoretical standpoint and are of paramount importance for calibrating the extragalactic distance scale. Type II Cepheids, on the other hand, are much more poorly understood due to their relative rarity and the fact that their pulsational behaviour is intrinsically more nonlinear, thus more complex.

In the HR diagram, RV Tauri stars and Type II Cepheids coexist with evolved massive Population I stars so it can be instructive to compare, for instance, a $6-M_{\odot}$ Type I Cepheid and an $0.6-M_{\odot}$ Type II Cepheid of similar effective temperature and luminosity. Having the same temperature and luminosity implies a similar structure with respect to the radial mass co-ordinate so that the driving mechanism of the stellar pulsation is similar. However, with respect to the radial depth co-ordinate their structure is very different, implying different pulsational characteristics (see Sasselov 1992 and references therein). Type I Cepheids tend to exhibit stable and regular pulsations which are readily understood and modelled. The pulsation modes for these stars are strongly confined for effective temperature and luminosity.

In contrast, Type II Cepheids tend to display unstable and complex pulsations. Their envelopes are unstable to many different pulsation modes over a wide range of effective temperature and luminosity. The explanation for this is based on the structure of the star: a low mass Type II star contains most of its mass in the core which is surrounded by a huge extended envelope of virtually zero density gradient. Any pulsations excited in such an envelope will be strongly nonadiabatic and nonlinear.

\section{MACHO Observations and Fourier Analysis}

The MACHO project has been described by Alcock et al. (1995). In this paper we generally refer to MACHO photometry $\left(V_{\mathrm{M}}\right.$ and $R_{\mathrm{M}}$ ) from 22 well-sampled fields concentrated along the bar of the LMC and covering a time span of up to seven years. Possible Type II Cepheid and RV Tauri star candidates were 
selected within specific ranges of period, brightness and colour. The light curves were searched for periodicities using a standard Fourier-type period finding code. The phased light and colour curves were then visually examined in order to classify the variables. The phased light curve of each Type II Cepheid or RV Tauri candidate was fitted with a truncated Fourier series and Fourier decomposition parameters were derived. For more details see Pollard et al. (1997) and Alcock et al. (1998).

Thirty-three Type II Cepheids and RV Tauri star candidates have been discovered. Finder charts, phased light and colour curves are shown in Pollard et al. (1997) and Alcock et al. (1998). In general it was found that Type II Cepheids with periods greater than $\sim 20 \mathrm{~d}$ display increasing variability in the depth, shape and phasing of their light curve minima. The periodograms of these stars also exhibit increasing strength of the subharmonic frequencies (or the double period) and the multiples of these frequencies. There has been some speculation that the RV Tauri stars may be undergoing period-doubled or possibly chaotic pulsations, and I will return to this point later.

When plotted on the double or 'formal' period, classic RV Tauri behaviour (Pollard et al. 1996), including alternation of the two minima (and maxima) is displayed by many of the longer period variables. In order to quantify these differences, Fourier decomposition of the phased light curves was undertaken. The most obvious trend is a general progression of $\Phi_{31}$ with increasing $\log P$. Unfortunately there appear to be no theoretical models for longer period $(P>$ 8d) Type II Cepheids for which Fourier decomposition parameters have been calculated, so we are unable to compare our results with theoretical predictions (for more details see Alcock et al. 1998).

\section{Absolute Luminosities and Period-Luminosity Relations}

Observations in the $V_{\mathrm{M}}$ and $R_{\mathrm{M}}$ bandpasses were converted to standard $V$ and $R$ magnitudes and mean dereddened $V$ and $R$ magnitudes and $V-R$ colours were calculated (Alcock et al. 1998). An LMC distance modulus of 18.5 was adopted in order to derive the absolute magnitudes of the LMC RV Tauri variables, giving $M_{V} \sim-4.5$ for variables with fundamental periods of about 50 days. This lends support to the proposition that these objects are luminous post-AGB stars.

The MACHO database of LMC photometry allows a direct interpretation of the luminosity for the Type II Cepheids and the RV Tauri stars and hence the $P-L$ and $P-L-C$ relations for these variables. The $P-L$ relation:

$$
M_{V}=(+1.34 \pm 0.45)-(3.07 \pm 0.35) \log P
$$

was derived, valid for $0.9<\log P<1.75$, with $\sigma=0.44$. The possibility that a colour effect was also present was investigated by including a colour term in the multivariate regression analysis. The $P-L-C$ relation thus derived was:

$$
M_{V}=(-0.61 \pm 0.20)-(2.95 \pm 0.12) \log P+(5.49 \pm 0.37)\left\langle(V-R)_{0}\right\rangle
$$

valid for $0.9<\log P<1.75$ with a $\sigma$ of 0.15 . The RV Tauri stars thus appear to be a direct extension of the Type II Cepheids to longer periods and these Population II variables are more accurately described by a $P-L-C$ relation 


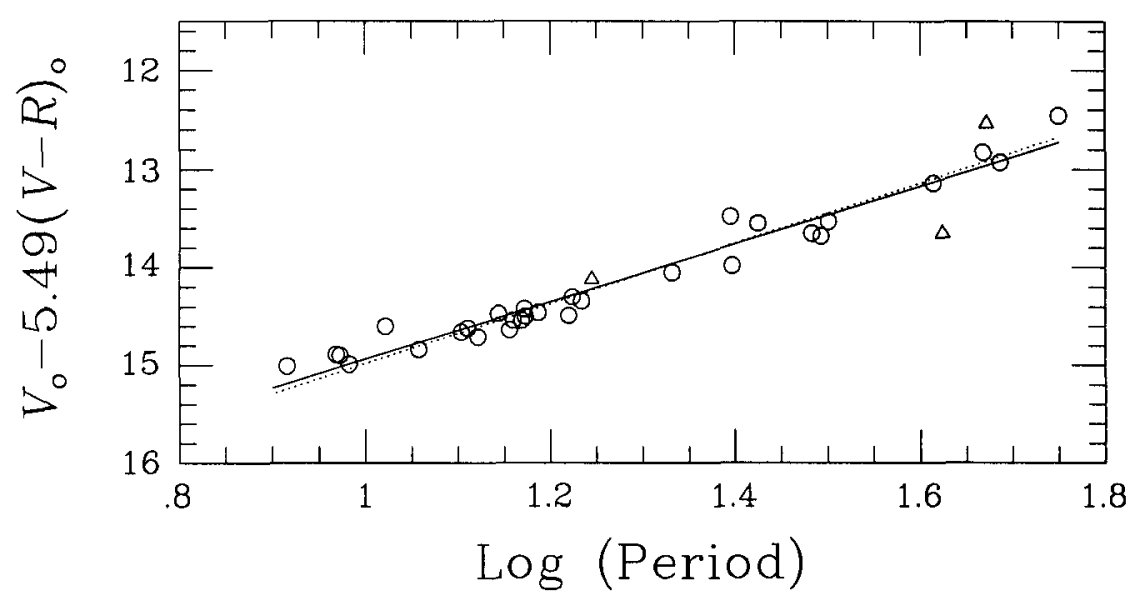

Figure 1. $P-L-C$ relation for the Type II Cepheids and RV Tauri stars in the LMC (solid line) using the single or fundamental period. The inverse regression relation is indicated with the dotted line. Triangles indicate stars omitted in deriving the $P-L-C$ relation.

than a $P-L$ relation (Fig. 1). The continuity of behaviour and properties is strong evidence that these two classes of star are related.

\section{Period Doubling and Chaotic Pulsations}

We now return to a point raised earlier, that the RV Tauri star may be part of a sequence which progresses from periodic to chaotic pulsators. Theoretical models (Buchler \& Kovács 1987; Kovács \& Buchler 1988; Aikawa 1993) of low-mass, high-luminosity, metal-deficient variables can show a sequence of behaviour from strictly periodic through to chaotic pulsations as the control parameter (usually $T_{\text {eff }}$ or $L$ ) is varied. At moderate $L / M$ ratios, the transition to chaos passes through a period-doubling bifurcation (or Feigenbaum sequence) whereas at higher $L / M$ ratios there is a more abrupt transition to chaos through the tangent bifurcation process (also known as 'intermittency'). The Fourier amplitude spectra for the period-doubled chaotic models show characteristic peaks at the subharmonic frequency and multiples of the subharmonic frequency, providing a possible test for analysing our observational data.

With respect to these theoretical predictions, at moderate $L / M$ ratios the Type II Cepheids would appear as periodic variables while the RV Tauri stars would appear to be in the period-doubling regime. Semiregular or irregular variables would be stars in the chaotic regime. For high $L / M$ ratios, the Type II Cepheids would once again be in the periodic regime while the rarer RV Tauri stars would be those stars that bracket the abrupt transition from periodic to chaotic, sometimes pulsating in a regular manner while appearing chaotic at other times. Both these scenarios are not inconsistent with the photometric variability that we observe in Type II Cepheids and RV Tauri stars. 


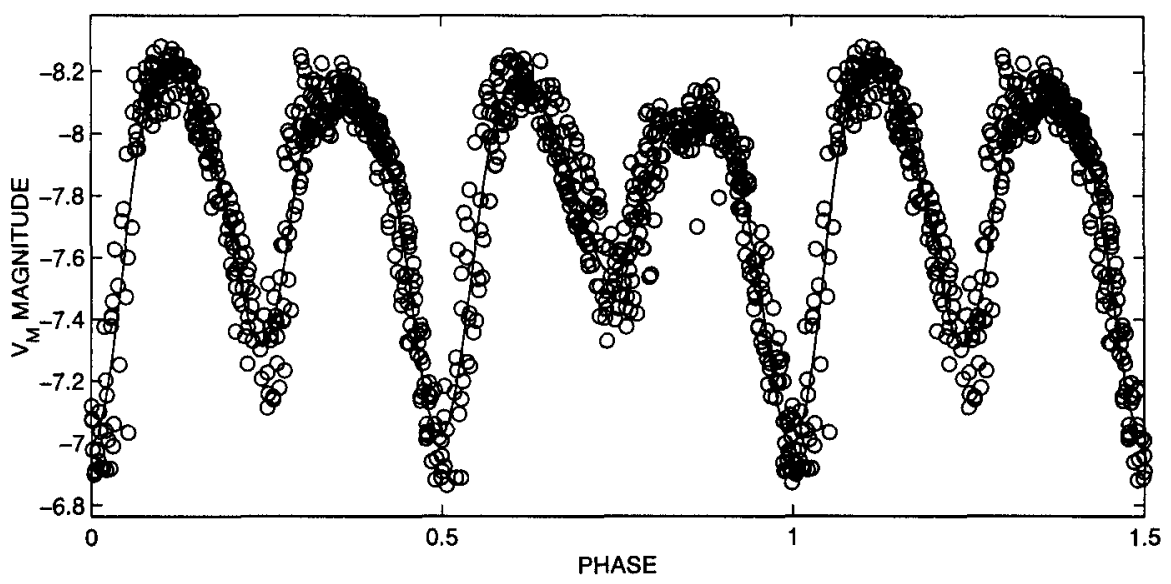

Figure 2. The light curve for the LMC RV Tauri star MACHO*05:37:45.0-69:54:16 ('fundamental' period $=30.408 \mathrm{~d}$ ) phased using the $P \times 4$ periodicity.

A recent analysis of AAVSO data for the galactic RV Tauri star R Sct (Buchler et al. 1995) has shown that the seemingly irregular pulsations in this star can be explained by a chaotic dynamic with an embedding dimension of 4 . This low dimension suggests that the complex pulsational behaviour in this star is the result of the nonlinear interaction of just two vibrational modes.

The quality and length of the time-series MACHO photometry allows an investigation of the stability of the pulsations in the LMC RV Tauri variables. The amplitude spectra for the Type II Cepheids and RV Tauri stars display a progression in behaviour with increasing period. The shorter period stars have amplitude spectra dominated by single strong frequencies, sometimes with multiples of the fundamental frequency if the light curve shows strong asymmetry. As the period of the star increases we see the appearance and increasing strength of subharmonic spikes (and their multiples) as the double period becomes more dominant. The cooler, more luminous RV Tauri variables show the most complex amplitude spectra.

A preliminary analysis of the long time-series MACHO photometry shows that one particular star (MACHO*05:37:45.0-69:54:16) has an obvious 'periodquadrupled' (or $P \times 4$ ) periodicity indicated in its amplitude spectrum and phased light curve (Fig. 2). This is supporting evidence for the period-doubling bifurcation transition to chaotic pulsations.

\section{Conclusions}

A study of the MACHO project LMC variable star database has identified thirtythree Type II Cepheids and RV Tauri stars candidates. The derived absolute magnitudes of the new LMC RV Tauri variables lends strong support to the proposition that these objects are luminous stars evolving off the AGB. A progression in behaviour from the short period Type II Cepheids through to the 
longer period RV Tauri stars in terms of their light and colour curve properties was noted. The variables with periods greater than about $20 \mathrm{~d}$ show increasingly strong RV Tauri characteristics.

A single $P-L-C$ relation is seen to describe both the Type II Cepheids and the RV Tauri stars in the LMC. The continuity in behaviour and properties is strong evidence that these two classes of variables are related.

A study of the amplitude spectra of these stars has shown the presence and increasing importance of subharmonic spikes (and their multiples) for the longer period variables. One particular star in our sample (MACHO*05:37:45.0 69:54:16) shows an obvious 'period-quadrupled' periodicity in its phased light curve, which is supporting evidence for the period-doubling bifurcation transition to chaotic pulsations.

\section{Future Work}

A number of refinements and extensions to the work summarised above should be undertaken. A larger sample of stars is required in order to examine the Fourier decomposition parameters and $P-L$ and $P-L-C$ relations for these stars in a more statistically significant manner. This may be achieved in a number of ways: the number of fields analysed in the LMC can be increased; the colour range should be extended in order to sample a larger range of effective temperatures; and the period range should be extended in order to search for other related stars. Finally, the above analysis is being extended to the MACHO SMC fields in order to search for and study Type II Cepheids and RV Tauri stars in a different metallicity environment.

Acknowledgments. During this research KRP was supported by a New Zealand Science and Technology Postdoctoral Fellowship and the Department of Physics and Astronomy, University of Canterbury. We are grateful for the skilled support by the technical staff at the Mt Stromlo Observatory. Work at: LLNL is supported by DOE contract W7405-ENG-48; the Center for Particle Astrophysics, UC campuses is supported by NSF grants AST 88-09616 and AST 91-20005; MSSSO is supported by the Australian Department of Industry, Technology, and Regional Development.

\section{References}

Aikawa, T. 1993, MNRAS, 262, 893

Alcock, C., Allsman, R. A, Axelrod, T. S., et al. 1995, AJ, 109, 1653

Alcock, C., Allsman, R. A., Alves, D. R., et al. 1998, AJ, 115, 1921

Buchler, J. R. \& Kovács, G. 1987, ApJ, 320, L57

Buchler, J. R., Serre, T., Kolláth, Z., \& Mattei, J. 1995, Phys.Rev.Lett., 73, 842

Kovács, G. \& Buchler, J. R. 1988, ApJ, 334, 971

Pollard, K. R., Alcock, C., Alves, D. R., et al. 1997, in Proc. 12th IAP Astrophys. Coll., Variable Stars and the Astrophysical Returns of Microlensing Surveys, ed. R. Ferlet, J.-P. Maillard, \& B. Raban (Gif-sur-Yvette: Edition Frontières), 219 
Pollard, K. R., Cottrell, P. L., Kilmartin, P. M., \& Gilmore, A. C. 1996, MNRAS, 279,949

Sasselov, D. D. 1993, in Proc. IAU Symp. 155, Planetary Nebulae, ed. R. Weinberger \& A. Acker (Dordrecht: Kluwer), 259

\section{Discussion}

Don Kurtz: In several of the light curves you showed the data on some cycles drops dramatically below the fit. Is this a real effect?

Karen Pollard: Yes, these are semiregular stars and show large amplitude variations (plus some phase variations too).

David Laney: What percentage of the Payne-Gaposchkin list of Type II Cepheids and RV Tauri stars is indeed in your sample from the MACHO database? Will these be in the outer fields not yet looked at?

Karen Pollard: Six of the 33 Type II Cepheids and RV Tauri stars found by me in the MACHO database are also in the Payne-Gaposchkin list. I do intend to look for the additional stars in the Payne-Gaposchkin list, and many of these should be in the outer fields.

John Hearnshaw: In future would you like to make use of the MOA database, especially given that MACHO will soon stop collecting data?

Karen Pollard: Yes.

Géza Kovács: 1. I think sequential prewhitening of the frequency spectra would be very vital for finding higher sub-harmonic components.

2. Checking the occurrence of period doubling at (roughly) fixed luminosity level as we move along the instability strip would be of foremost importance.

Karen Pollard: 1. Prewhitening would be extremely problematic with these stars which are semiregular and show varying amplitudes and/or phases.

2. Yes, this is a good idea and would be helped by increasing the sample size of Type II Cepheids and RV Tauri stars in the LMC, which I also hope to do in the near future. 